\title{
Forecasting Inflation Using Univariate Continuous-Time Stochastic Models
}

\author{
K. Fergusson* \\ The University of Melbourne, Victoria 3010, Australia
}

November 26, 2018

\begin{abstract}
Inflation rate forecasting is of much use in economics, insurance and pensions. For example, economic policy makers need forecasts to monitor and adjust policy settings to contain excessive inflation. Inflation rate forecasts can be used as part of wage negotiations so that employees' purchasing powers are maintained. Inflation-linked securities such as treasury income protected securities (TIPS) require inflation forecasts to value them. Actuaries in insurance companies set inflation rate assumptions in their pricing of insurance products. Additionally, actuaries require forecasts of inflation in valuing the inflation-linked liabilities of pension funds. We investigate the applicability of several continuous-time stochastic models to forecasting inflation rates out to twenty years. The benchmark for univariate inflation forecasts is the one-year inflation rate, as highlighted by Atkeson and Ohanian in their 2001 paper, otherwise known at the random walk model. We perform a comprehensive analysis of the forecast performance of the considered continuous time stochastic models of the consumer price inflation index and find that over longer forecasting horizons, i.e. those beyond five years, the lognormal index model having Ornstein-Uhlenbeck drift rate provides the best forecasts.
\end{abstract}

Keywords: Maximum likelihood estimation, lognormal model, minimal market model, Vasicek model, inflation rate, squared Bessel process, modified Bessel function of the first kind.

JEL Classification: C02, C13.

\section{Introduction}

Inflation rate forecasting is of much use in economics, insurance and pensions. For example, economic policy makers need forecasts to monitor and adjust policy settings to contain excessive inflation. Inflation rate forecasts can be used as

${ }^{*}$ This research is supported by an Australian Government Research Training Program Scholarship. 
part of wage negotiations so that employees' purchasing powers are maintained. Inflation-linked securities such as treasury income protected securities (TIPS) require inflation forecasts to value them. Actuaries in insurance companies set inflation rate assumptions in their pricing of insurance products. Additionally, actuaries require forecasts of inflation in valuing the inflation-linked liabilities of pension funds.

As varied are the purposes, forecasting inflation has many approaches, for example as described in Meyer and Pasaogullari [2010], which incorporate into the forecasting model such information as inflation expectations surveys, Phillips curves, import price indices, wage indices, commodity prices and interest rates. A simpler approach involves only the inflation time series itself in computing forecasts.

Along these simpler lines, the focus of this paper is on forecasts based on continuous-time univariate stochastic processes. Continuous-time stochastic processes, described by stochastic differential equations (SDEs), can be viewed as limiting cases of discrete-time stochastic processes, described by discrete time series. While the tractability of continuous-time SDEs is limited to a few classes of models, their parsimony allows precise fitting of model parameters via maximum likelihood estimation; see for example Fergusson and Platen [2015] and Fergusson [2017]. In contrast, ARIMA and GARCH models can contain many parameters, potentially leading to over-fitting of the models and poor forecasting performances out-of-sample, and their parameter estimation times are correspondingly longer. Additionally, GARCH and its variations are only able to capture some types of heteroskedastic behaviour; see, for example, Bergstrom [1984].

Typically inflation forecasts based only a single inflation series employ time series models such as autoregressive integrated moving average (ARIMA) models and nonlinear or time-varying univariate models such as GARCH. Among the simplest of these is the random walk model proposed in Atkeson and Ohanian [2001], in which the forecast of the four-quarter rate of inflation is the average quarterly inflation rate over the previous four quarters. These univariate models can also be extended to include as predictors one or more alternative inflation measures. A more recent univariate model is the unobserved components-stochastic volatility (UC-SV) model, given in Stock and Watson [2007], in which the quarterly inflation rate $\pi_{t}$ has a stochastic trend $\tau_{t}$, a serially uncorrelated disturbance $\eta_{t}$, and stochastic volatility $\sigma_{t}$. The UC-SV has become the benchmark model for univariate forecasting of inflation and we investigate a continuous-time model which is a variation of this, which we call the unobserved components-constant volatility (UC-SV) model.

In this paper we supply six continuous-time stochastic models, the second of which embodies the random walk model of Atkeson and Ohanian [2001] and the remaining of which are variations of this. We show that our final model can be viewed as a continuous-time version of the UC-CV model. In Section 2 we describe our inflation data and identify some stylised features which we aim to capture in our models. In Section 3 we describe the models. In Section 4 we give details on how the parameters of the models are estimated. In Section 5 we 


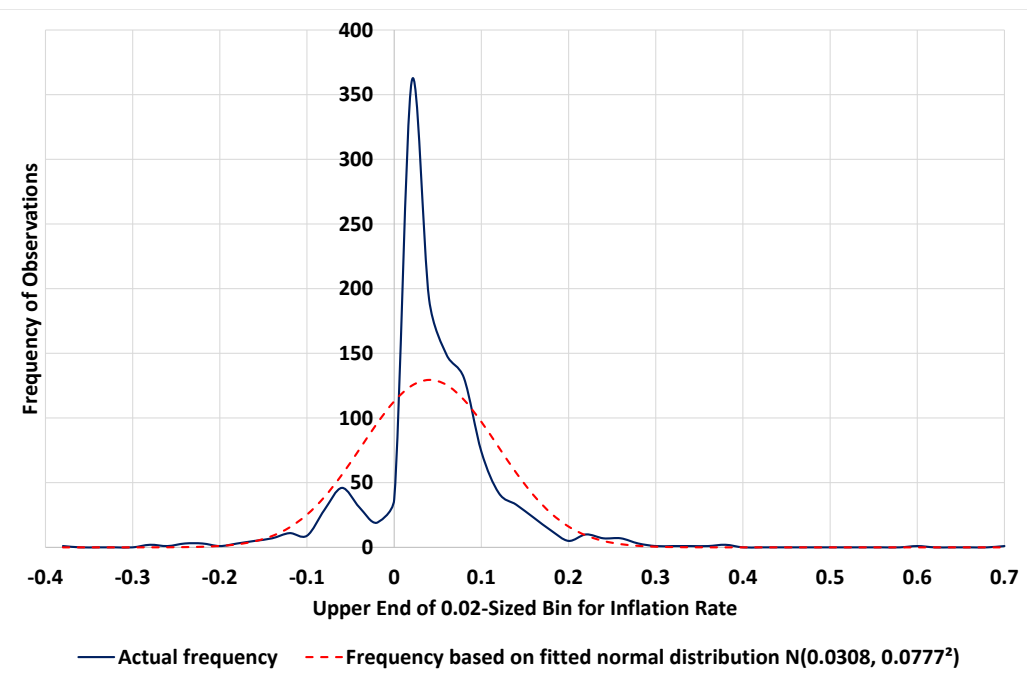

Figure 1: Histogram of continuously compounded monthly inflation rates, annualised, using 0.02-sized bins (1913-2018).

present the results of out-of-sample forecasting with the models and Section 6 concludes.

\section{Data and Motivation}

The data set employed for our analysis is the monthly series of the U.S. Bureau of Labor Statistics, Consumer Price Index for All Urban Consumers: All Items [CPIAUCNS], retrieved from FRED, Federal Reserve Bank of St. Louis; https://fred.stlouisfed.org/series/CPIAUCNS. The series is not seasonally adjusted and commences in January 1913 and ends in July 2018. Our choice is motivated purely by the desire to have a long data series for our estimation of model parameters. Of course the composition of the index and the economic monetary and fiscal policies concerning inflation have changed over the past century. However, we seek to model the dynamics of the CPI as much as possible using the models given in Section 3.

The histogram of monthly inflation rates is shown in Figure 1 where the corresponding histogram of the fitted normal distribution is overlaid. It is clear that the distribution of actual inflation rates is more peaked and fatter-tailed than the normal distribution and therefore a good model ought to capture this stylised feature. 
We assume that our index data points are recorded at the $n+1$ equidistant times $t_{0}, t_{1}, \ldots, t_{n}$, with spacing $\Delta$ measured in years.

\section{Continuous-Time Stochastic Models}

We discuss the models employed in our forecasting. Each model is specified as a stochastic differential equation (SDE) of the consumer price index, which we denote by $I_{t}$ at time $t$, on a filtered probability space $\left(\Omega, \underline{\mathcal{F}},\left(\mathcal{F}_{t}\right)_{t \geq 0}, P\right)$ satisfying the usual conditions. The models employed are the lognormal model, the lognormal model having time-varying drift rate, the square root model, the $3 / 2$ model, the Vasicek inflation rate model and the lognormal model with Vasicek drift rate.

\subsection{Lognormal Model}

The first is the lognormal model, used in Jarrow and Yildirim [2003] for pricing treasury inflation protected securities (TIPS),

$$
d I_{t}=\pi I_{t} d t+\sigma I_{t} d W_{t}
$$

where $W$ is a Wiener process adapted to the filtration $\left(\mathcal{F}_{t}\right)_{t \geq 0}$, the constant $\pi$ is the drift, and $\sigma>0$ is the diffusion coefficient. One implication of using this model is that the quadratic variation of the logarithm of $I_{t}$ is equal to $\sigma^{2} t$, for $t \geq 0$. The empirically computed quadratic variation is shown as the solid line in Figure 2, where it is approximated by a piecewise linear function over the periods 1913 to 1949 and 1950 to 2018, This is consistent with the linear quadratic variation implied by the lognormal model. We can solve (1) giving

$$
d \log I_{t}=\pi d t+\sigma d W_{t},
$$

so that the logarithm of inflation is a random walk with trend. This also reveals another implication of (1), which is that log-returns of the index are normally distributed, and this is not reflected in the histogram of actual log-returns in Figure 1.

\subsection{Lognormal Model with Time-Varying Drift Rate}

The second inflation model considered is the lognormal model with time-varying drift rate, as specified by the SDE

$$
d I_{t}=\pi_{t} I_{t} d t+\sigma I_{t} d W_{t}
$$

where the instantaneous inflation rate $\pi_{t}$ is unobserved and is inferred by applying a filter, as described in Section 4.2. This model closely corresponds to the model in Atkeson and Ohanian [2001]. 


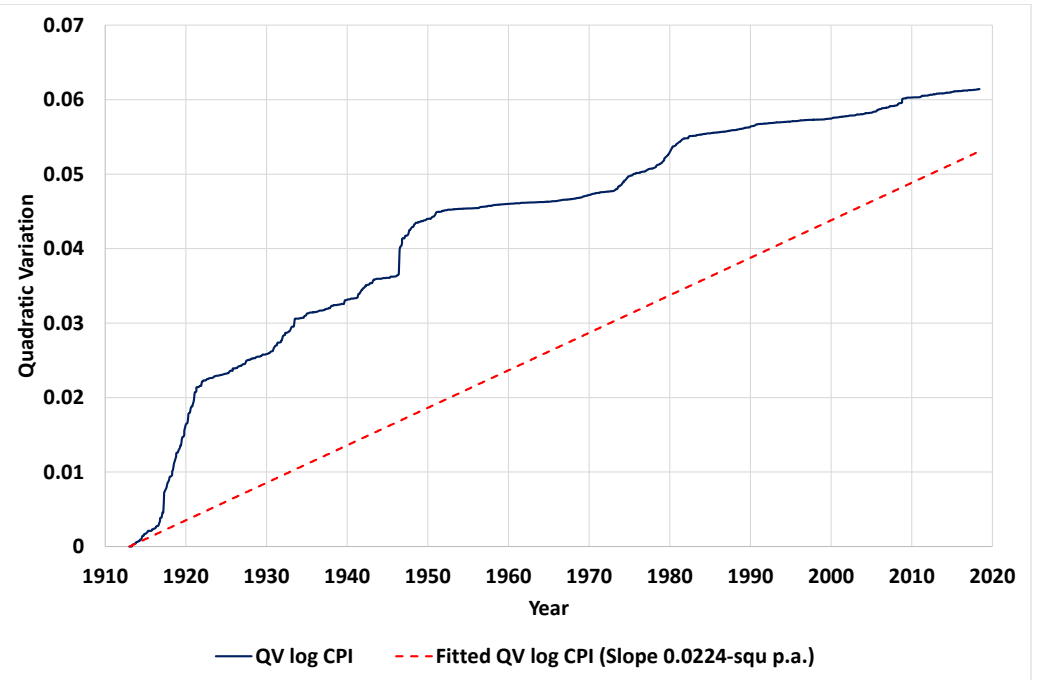

Figure 2: Empirically computed and theoretical quadratic variations of the logarithm of the CPI. 


\subsection{Square Root Model}

The third model is the square root model, so named because its diffusion coefficient is proportional to the square root of the index. Coined the minimal market model, it was first used in Platen [2001] for modelling the discounted stock index, but is adapted here to model inflation,

$$
d I_{t}=\alpha_{t} d t+\sqrt{\alpha_{t} I_{t}} d W_{t},
$$

where $\alpha_{t}=\alpha_{0} \exp (\eta t)$ is the growth of inflation, $\alpha_{0}$ is a positive constant and $\eta$ is the inflation growth rate. Now we define the quadratic variation of the square root of $I_{t}$ as

$$
[\sqrt{I}]_{t}=\lim _{\Delta \rightarrow 0} \sum_{i=1}^{n}\left(\sqrt{I_{t_{i}}}-\sqrt{I_{t_{i-1}}}\right)^{2}
$$

For the process in (4) it is equal to

$$
\varphi_{t} \equiv[\sqrt{I}]_{t}=[\sqrt{I}]_{0}+\frac{1}{4} \int_{0}^{t} \alpha_{u} d u=\frac{1}{4} \alpha_{0}(\exp (\eta t)-1) / \eta,
$$

for $t \geq 0$, and we denote this by $\varphi_{t}$. The empirically computed quadratic variation, using the formula

$$
[\sqrt{I}]_{t} \approx \sum_{i=1}^{n}\left(\sqrt{I_{t_{i}}}-\sqrt{I_{t_{i-1}}}\right)^{2}
$$

is shown in Figure 3. Overlaid is the theoretical quadratic variation, using (6) and the parameter estimates given in Table 1. It is apparent that the theoretical quadratic variation is a good fit to the empirically computed quadratic variation.

\section{$3.4 \quad 3 / 2$ Model}

The fourth model is the $3 / 2$ model, so named because its diffusion coefficient is proportional to the square root of the cube of the index. It has been proferred in Cox et al. [1985b] for modelling the inflation rate. For modelling the short rate, this model was developed in Platen [1999], and subsequently given in Ahn and Gao [1999]. It has also been used for modelling the variance rate of stock indices; see for example Platen [2001]. However, it is adapted here to model the inflation index,

$$
d I_{t}=\sqrt{\alpha_{t} I_{t}^{3}} d W_{t}
$$

where $\alpha_{t}=\alpha_{0} \exp (\eta t)$ is the growth of inflation, $\alpha_{0}$ is a positive constant and $\eta$ is the inflation growth rate. Now we define the quadratic variation of the reciprocal of the square root of $I_{t}$ as

$$
[1 / \sqrt{I}]_{t}=\lim _{\Delta \rightarrow 0} \sum_{i=1}^{n}\left(1 / \sqrt{I_{t_{i}}}-1 / \sqrt{I_{t_{i-1}}}\right)^{2}
$$




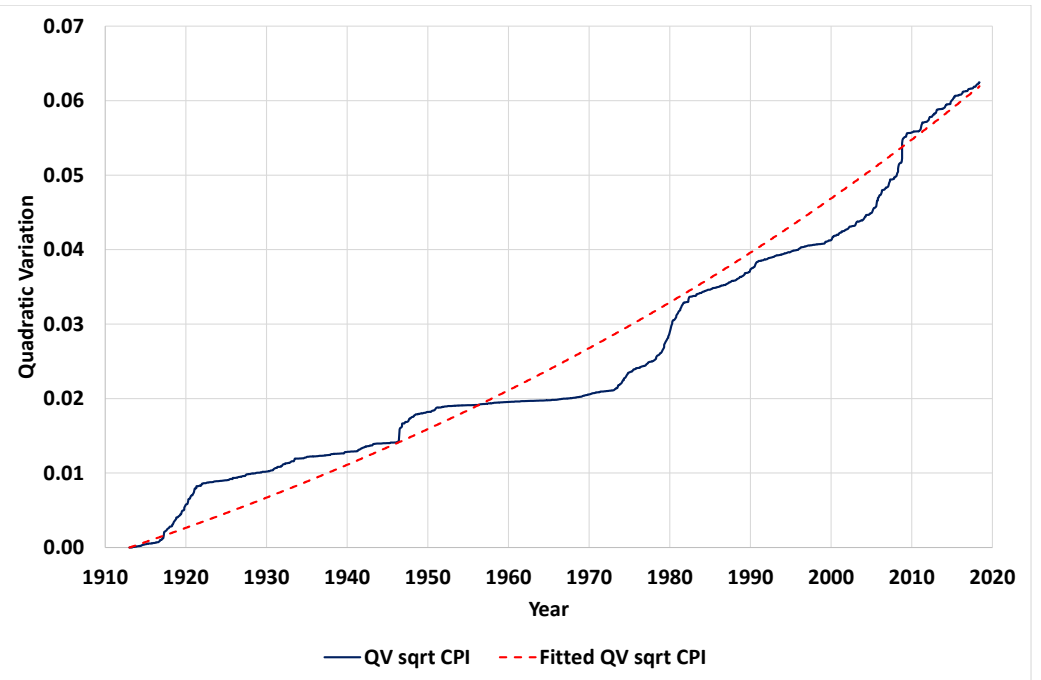

Figure 3: Empirically computed and theoretical quadratic variations of the square root of the CPI. 


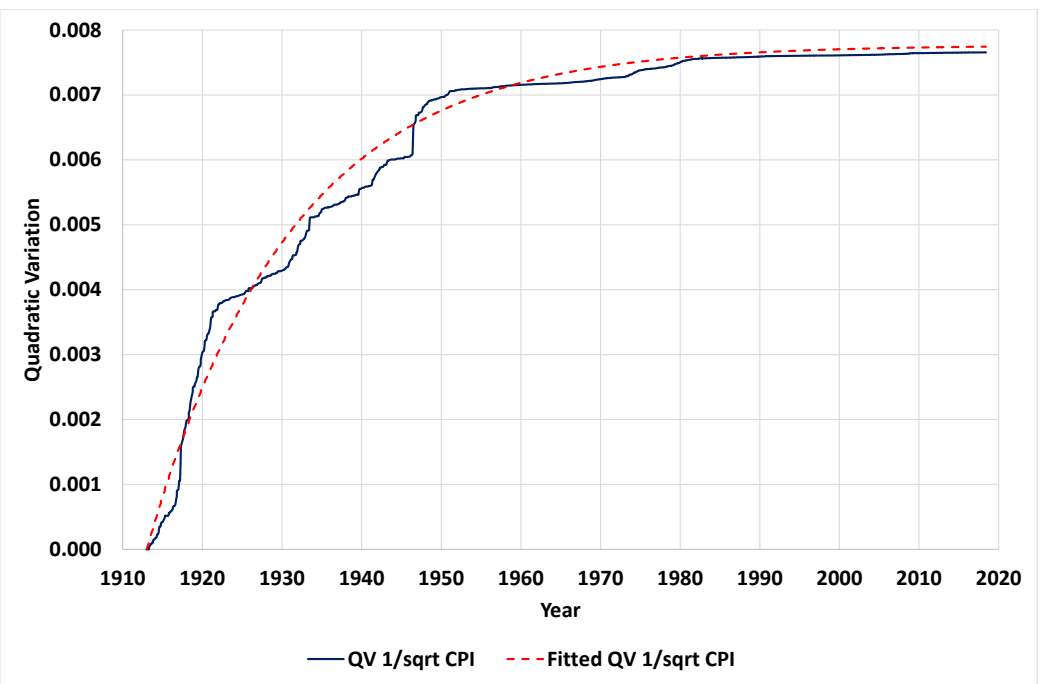

Figure 4: Empirically computed and theoretical quadratic variations of the reciprocal of the square root of the CPI.

For the process in (8) it is equal to

$$
\xi_{t} \equiv[1 / \sqrt{I}]_{t}=[1 / \sqrt{I}]_{0}+\frac{1}{4} \int_{0}^{t} \alpha_{u} d u=\frac{1}{4} \alpha_{0}(\exp (\eta t)-1) / \eta,
$$

for $t \geq 0$, and we denote this by $\xi_{t}$. The empirically computed quadratic variation, using the formula

$$
[1 / \sqrt{I}]_{t} \approx \sum_{i=1}^{n}\left(1 / \sqrt{I_{t_{i}}}-1 / \sqrt{I_{t_{i-1}}}\right)^{2}
$$

is shown in Figure 4. Overlaid is the theoretical quadratic variation, using (10) and the parameter estimates given in Table 1. It is apparent that the theoretical quadratic variation is a good fit to the empirically computed quadratic variation.

\subsection{Vasicek Inflation Rate Model}

The fifth model is the Vasicek model as given in Vasicek [1977] for modelling the short rate, but is applied here to modelling the inflation rate $\pi_{t}$, specified by the SDE

$$
d I_{t}=\pi_{t} I_{t} d t
$$


where the instantaneous inflation rate $\pi_{t}$ obeys the SDE

$$
d \pi_{t}=\kappa\left(\bar{\pi}-\pi_{t}\right) d t+\omega d Z_{t} .
$$

There are other candidate short rate models such as the CIR short rate model given in Cox et al. [1985a] and the 3/2 model given in Ahn and Gao [1999], but they avoid the possibility of negative rates. It is because negative inflation rates are possible under the Vasicek model that it is being considered in this paper.

\subsection{Lognormal Model with Vasicek Drift Rate}

The sixth model is that given in Chiarella et al. [2007],

$$
d I_{t}=\pi_{t} I_{t} d t+\sigma I_{t} d W_{t}
$$

where the instantaneous inflation rate $\pi_{t}$ obeys the SDE

$$
d \pi_{t}=\kappa\left(\bar{\pi}-\pi_{t}\right) d t+\omega d Z_{t},
$$

for a Wiener process $Z$ which we assume to be independent of $W$ and where $\bar{\pi}$, $\kappa, \omega$ are constants.

Two variations of this sixth model are proferred in Cox et al. [1985a], where the inflation rate SDE has either a square root or $3 / 2$ exponent for $\pi_{t}$ in the diffusion coefficient and where the CPI SDE has a factor equal to square root of $i_{t}$ inserted in its diffusion term. However, implementing these models requires that the unobserved inflation rates are all positive, which we cannot guarantee for our CPI data set. The quadratic variation of $\pi_{t}$ given in our data set is shown in Figure 6, which is approximately piecewise linear over the two periods 1913 to 1949 and 1950 to 2018 and is therefore consistent with the linear quadratic variation of $i_{t}$ given in (15). Furthermore, (15) captures the mean-reverting behaviour of $\pi_{t}$, illustrated in Figure 5 .

\section{Parameter Estimation}

The estimated parameters are found using maximum likelihood estimation and are given in Table 1. We describe the methodologies in arriving at our maximum likelihood estimates. Standard errors are obtained as square roots of the diagonal elements of the reciprocal of the Fisher information matrix.

\subsection{Lognormal Model}

The maximum likelihood estimates of the parameters in (1) are straightforwardly estimated from the sample mean and variance of increments of the log- 


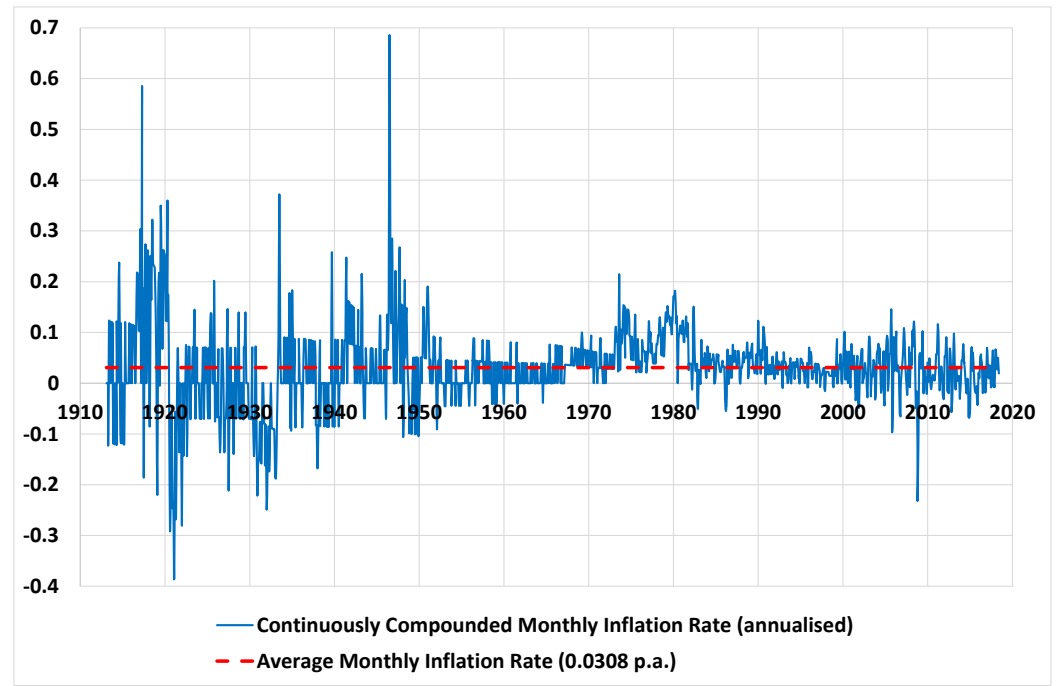

Figure 5: Inflation rate history (1913-2018).

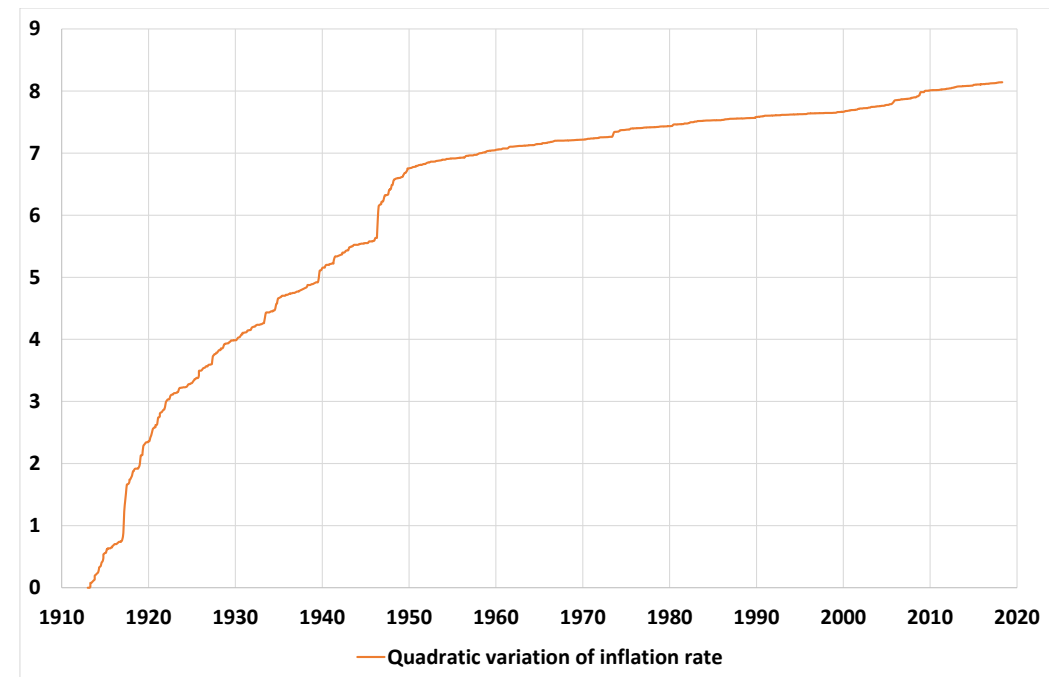

Figure 6: Empirically computed quadratic variation of the inflation rate. 
arithm of the CPI. We have

$$
\begin{aligned}
\hat{\pi} & =\frac{1}{n \Delta} \sum_{i=1}^{n} \log \left(I_{t_{i}} / I_{t_{i-1}}\right)+\frac{1}{2} \hat{\sigma}^{2} \\
\hat{\sigma^{2}} & =\left[\frac{1}{n} \sum_{i=1}^{n}\left\{\log \left(I_{t_{i}} / I_{t_{i-1}}\right)\right\}^{2}-\left\{\frac{1}{n} \sum_{i=1}^{n} \log \left(I_{t_{i}} / I_{t_{i-1}}\right)\right\}^{2}\right] / \Delta .
\end{aligned}
$$

\subsection{Lognormal Model with Local Drift Rate}

The log-likelihood function is

$$
\ell(\sigma)=\sum_{i=1}^{n}\left\{\frac{1}{\sqrt{2 \pi \sigma^{2} \Delta}} \frac{1}{I_{t_{i}}} \exp \left\{-\frac{1}{2} \frac{\left(\log I_{t_{i}}-\log I_{t_{i-1}}-\left(\pi_{t_{i-1}}-\frac{1}{2} \sigma^{2}\right) \Delta\right)^{2}}{\sigma^{2} \Delta}\right\}\right\}
$$

where we infer the unobserved rates by applying a filter $f$ to the observed noisy logarithms of the index $\log \left(I_{t_{j}}\right), j \in\{i-11, i-10, \ldots, i\}$, and computing $\pi_{t_{i}}$ as the first-order derivative of $f$ at time $t_{i}$. The filter $f$ is obtained by an exponentially weighted regression of $\log \left(I_{t_{j}}\right)$ on the basis terms of the quadratic $f\left(t_{j}\right)=a+b t_{j}+c t_{j}^{2}$ for $j \in\{i-11, i-10, \ldots, i\}$, from which the drift rate is given by $\pi_{t_{i}}=\left.\frac{d f(t)}{d t}\right|_{t=t_{i}}=b+2 c t_{i}$.

Before stating the theorem which provides the maximum likelihood estimate of $\sigma$, we give a useful lemma.

Lemma 1 Let $f$ be a function defined on $(0, \infty)$ as

$$
f(v)=\log v+\frac{A}{v}+B v,
$$

where $A$ and $B$ are positive constants. Then $f$ has a global miminum on $(0, \infty)$ at

$$
v^{*}=\{\sqrt{A+1 /(4 B)}-\sqrt{1 /(4 B)}\} / \sqrt{B} .
$$

Proof. For $v>0$ we have

$$
\begin{aligned}
f(v) & =f\left(v^{*}\right)+\int_{v^{*}}^{v}\left(\frac{1}{t}-\frac{A}{t^{2}}+B\right) d t \\
& =f\left(v^{*}\right)+\int_{v^{*}}^{v} \frac{1}{t^{2}}\left(B t^{2}+t-A\right) d t \\
& =f\left(v^{*}\right)+\int_{v^{*}}^{v} \frac{1}{t^{2}}\left\{(\sqrt{B} t+\sqrt{1 /(4 B)})^{2}-(A+1 /(4 B))\right\} d t,
\end{aligned}
$$

where $v^{*}$ is given in (19). For $t>v^{*}$ the integrand is positive, so that for $v>v^{*}$, $f(v)>f\left(v^{*}\right)$. Also, for $t<v^{*}$ the integrand is negative, so that for $v<v^{*}$, the integral can be rewritten as

$$
\int_{v}^{v^{*}}-\frac{1}{t^{2}}\left\{(\sqrt{B} t+\sqrt{1 /(4 B)})^{2}-(A+1 /(4 B))\right\} d t
$$


which is positive, and thus $f(v)>f\left(v^{*}\right)$.

Theorem 2 The maximum likelihood estimate of $\sigma$ is given by

$$
\hat{\sigma}^{2}=\frac{2}{\Delta}\left[-1+\sqrt{1+\frac{1}{n} \sum_{i-1}^{n}\left(\log I_{t_{i}}-\log I_{t_{i-1}}-\pi_{t_{i-1}} \Delta\right)^{2}}\right] .
$$

Proof. We rewrite the log-likelihood function in (17) as

$$
\ell(\sigma)=-\frac{n}{2}\left\{\log (2 \pi)+\frac{2}{n} \sum_{i=1}^{n} \log \left(I_{t_{i}}\right)+\frac{1}{n} \sum_{i=1}^{n}\left(\log I_{t_{i}}-\log I_{t_{i-1}}-\pi_{t_{i-1}} \Delta\right)+f\left(\sigma^{2} \Delta\right)\right\},
$$

where $f$ is given (18),

$$
A=\frac{1}{n} \sum_{i-1}^{n}\left(\log I_{t_{i}}-\log I_{t_{i-1}}-\pi_{t_{i-1}} \Delta\right)^{2}
$$

and $B=\frac{1}{4}$. From the preceding lemma, $f$ achieves a global minimum when $\sigma^{2} \Delta$ is

$$
\sigma^{2} \Delta=2\{\sqrt{A+1}-1\}
$$

and the result follows.

\subsection{Square Root Model}

The parameters in (4) are estimated using the formulae given in Theorem 2 in Fergusson [2017], which are

$$
\begin{aligned}
\hat{\bar{\alpha}}_{0, \Delta} & \approx \frac{1}{n} \sum_{i=1}^{n} \frac{\left(\sqrt{I_{t_{i}}}-\sqrt{I_{t_{i-1}}}\right)^{2}}{\frac{1}{4 \eta}(\exp (\eta \Delta)-1) \exp (\eta(i-1) \Delta)} \\
\hat{\eta}_{\Delta} & \approx \frac{6}{\Delta(n+1)}\left[\frac{1}{\left(\begin{array}{c}
n \\
2
\end{array}\right)} \sum_{i \in K}(i-1) \log \left\{\left(\sqrt{I_{t_{i}}}-\sqrt{I_{t_{i-1}}}\right)^{2}\right\}\right. \\
& \left.-\frac{1}{n} \sum_{i \in K} \log \left\{\left(\sqrt{I_{t_{i}}}-\sqrt{I_{t_{i-1}}}\right)^{2}\right\}\right],
\end{aligned}
$$

where summation is over the set $K=\left\{i=1,2, \ldots, n: I_{t_{i}} \neq I_{t_{i-1}}\right\}$ and where there are $n+1$ observations of the inflation index $I_{t}^{\delta_{*}}$, for $i=0,1,2, \ldots, n$.

\subsection{3/2 Model}

Because the dynamics of $1 / I$ obey the SDE in (4), the parameters in (8) are estimated using a straightforward modification to the formulae given in (26), 
resulting in

$$
\begin{aligned}
\hat{\bar{\alpha}}_{0, \Delta} & \approx \frac{1}{n} \sum_{i=1}^{n} \frac{\left(1 / \sqrt{I_{t_{i}}}-\sqrt{1 / I_{t_{i-1}}}\right)^{2}}{\frac{1}{4 \eta}(\exp (\eta \Delta)-1) \exp (\eta(i-1) \Delta)} \\
\hat{\eta}_{\Delta} & \approx \frac{6}{\Delta(n+1)}\left[\frac{1}{\left(\begin{array}{c}
n \\
2
\end{array}\right)} \sum_{i \in K}(i-1) \log \left\{\left(1 / \sqrt{I_{t_{i}}}-1 / \sqrt{I_{t_{i-1}}}\right)^{2}\right\}\right. \\
& \left.-\frac{1}{n} \sum_{i \in K} \log \left\{\left(1 / \sqrt{I_{t_{i}}}-1 / \sqrt{I_{t_{i-1}}}\right)^{2}\right\}\right]
\end{aligned}
$$

where summation is over the set $K=\left\{i=1,2, \ldots, n: I_{t_{i}} \neq I_{t_{i-1}}\right\}$ and where there are $n+1$ observations of the inflation index $I_{t}^{\delta_{*}}$, for $i=0,1,2, \ldots, n$.

\subsection{Vasicek Inflation Rate Model}

The parameters in (12) and (13) are estimated using the formulae given in Theorem 1 in Fergusson and Platen [2015], which are

$$
\begin{aligned}
\hat{\bar{\pi}} & =\frac{S_{1} S_{00}-S_{0} S_{01}}{S_{0} S_{1}-S_{0}^{2}-S_{01}+S_{00}}, \\
\hat{\kappa} & =\frac{1}{\Delta} \log \frac{S_{0}-\bar{\pi}}{S_{1}-\bar{\pi}}, \\
\hat{\omega}^{2} & =\frac{1}{n \beta\left(1-\frac{1}{2} \kappa \beta\right)} \sum_{i=1}^{n}\left(\pi_{t_{i}}-m_{t_{i-1}}\left(t_{i}\right)\right)^{2},
\end{aligned}
$$

where

$$
\begin{gathered}
m_{t_{i-1}}\left(t_{i}\right)=\bar{\pi} \kappa \beta+\pi_{t_{i-1}}(1-\kappa \beta), \\
S_{0}=\frac{1}{n} \sum_{i=1}^{n} \pi_{t_{i-1}}, \quad S_{1}=\frac{1}{n} \sum_{i=1}^{n} \pi_{t_{i}}, \\
S_{00}=\frac{1}{n} \sum_{i=1}^{n} \pi_{t_{i-1}} \pi_{t_{i-1}}, \quad S_{01}=\frac{1}{n} \sum_{i=1}^{n} \pi_{t_{i-1}} \pi_{t_{i}},
\end{gathered}
$$

and $\beta=\frac{1}{\kappa}(1-\exp (-\kappa \Delta))$.

Because the probability density of the inflation index $I_{t}$ conditional on the value of the inflation index $I_{t-\Delta}$ is

$$
\begin{aligned}
f_{I_{t} \mid I_{t-\Delta}}(x) & =\frac{1}{\sqrt{2 \pi V}} \frac{1}{x} \\
& \times \exp \left\{-\frac{1}{2} \frac{\left(\log (x)-\log \left(I_{t-\Delta}\right)-\pi_{t-\Delta} \beta-\bar{\pi}(\Delta-\beta)\right)^{2}}{V}\right\},
\end{aligned}
$$




\begin{tabular}{lll} 
CPI Model & Model Diagnostic & Parameter Estimates \\
\hline Lognormal & $\ell=2619.260616$ & $\hat{\pi}=0.031053(0.002186)$ \\
& $A I C=-5234.521231$ & $\hat{\sigma}=0.022439(0.000446)$ \\
& $B I C=-5224.235576$ & \\
\hline Lognormal with Local Drift & $\ell=2702.915264$ & $\hat{\sigma}=0.021003(0.000418)$ \\
& $A I C=-5403.830529$ & \\
& $B I C=-5398.687701$ & \\
\hline Square Root & $\ell=2647.742582$ & $\hat{\alpha_{0}}=0.001468(0.000107)$ \\
& $A I C=-5291.485163$ & $\hat{\eta}=0.008320(0.001155)$ \\
& $B I C=-5281.199508$ & \\
\hline $3 / 2$ & $\ell=2701.368083$ & $\hat{\alpha_{0}}=0.001715(0.000137)$ \\
& $A I C=-5398.736166$ & $\hat{\eta}=-0.055188(0.001312)$ \\
& $B I C=-5388.450512$ & \\
\hline Vasicek Drift & $\ell=2243.829436$ & $\hat{\pi}=0.030839(0.003630)$ \\
& $A I C=-4481.658873$ & $\hat{\kappa}=9.128286(0.633592)$ \\
& $B I C=-4466.230390$ & $\hat{\omega}=0.332236(0.009359)$ \\
\hline Lognormal with Vasicek Drift & $\ell=2729.923151$ & $\hat{\pi}=0.031122(0.006782)$ \\
& $A I C=-5451.846301$ & $\hat{\kappa}=1.745702(0.195865)$ \\
& $B I C=-5431.274992$ & $\hat{\omega}=0.121449(0.002592)$ \\
& & $\hat{\sigma}=0.019800(0.000424)$
\end{tabular}

Table 1: Parameter estimates of inflation rate models, with standard errors shown in brackets.

where

$$
V=\omega^{2}\left(\frac{\Delta}{\kappa^{2}}+\frac{1}{2 \kappa^{3}}-\frac{1}{2 \kappa}\left(\beta+\frac{1}{\kappa}\right)^{2}\right),
$$

the log-likelihood function, whose value is supplied in Table 1 , is

$\ell=\sum_{i=1}^{n}\left\{\log \frac{1}{\sqrt{2 \pi V}}-\log \left(I_{t_{i}}\right)-\frac{1}{2} \frac{\left(\log \left(I_{t_{i}}\right)-\log \left(I_{t_{i-1}}\right)-\pi_{t_{i-1}} \beta-\bar{\pi}(\Delta-\beta)\right)^{2}}{V}\right\}$.

\subsection{Lognormal Model with Vasicek Drift}

Estimation of the parameters of the Vasicek drift rate process, given in (15), is a straightforward application of the formulae given in Section 4.5, when the drift rates are known and observed. One approach to parameter estimation when drift rates are unobserved is undertaken in Chiarella et al. [2007], where a Kalman filter is used to infer the rates. The approach employed here is similar, where we infer the unobserved rates according the method given in Section 4.2. Thus, the parameters in (15) can be estimated using the formulae in Section 4.5; see Fergusson and Platen [2015] for further details. Finally, a maximum likelihood approach similar to that given Theorem 2 in Section 4.2 solves for $\sigma$ in (14). 


\section{$5 \quad$ Forecasting Inflation Rates}

As at time $t$, we seek a forecast of the one-year log return of the inflation index at time $T$, where $T>t$,

$$
\pi_{T-12 \Delta, T}=\frac{1}{12 \Delta} \log \left(I_{T} / I_{T-12 \Delta}\right)
$$

and a forecast of the one-year inflation rate at time $T$

$$
i_{T-12 \Delta, T}=\left(I_{T} / I_{T-12 \Delta}\right)^{1 /(12 \Delta)}-1 .
$$

In the light of empirical evidence given in Meyer and Pasaogullari [2010], we use the current one-year inflation rate, similar to that given in Atkeson and Ohanian [2001], as our reference forecast over all forecast horizons. In Figure 7 we present the results of out-of-sample forecasts of annual inflation rates over varying forecasting horizons for each of the models. The best models over fiveyear forecast horizons are the lognormal, lognormal Vasicek and the Vasicek with drift models. In Figure 8 we show the root-mean-squared errors (RMSEs) of the one-year-ahead forecasts over each half decade over the past fifty years, where it is evident that the periods in the 1970s had the highest RMSEs. Also, the AO model remains the best model for forecasting one-year-ahead inflation rates. In Figure 9 we show the scatter plot of the forecasts versus the actual one-year inflation rate over the past fifty years, where it is evident that the OA model has the best explanatory power, having the highest R-squared.

\subsection{Lognormal Model}

Lemma 3 Our forecast of the log return of the inflation index under the lognormal model is

$$
E\left(\pi_{T-\Delta, T} \mid \mathcal{F}_{t}\right)=\pi-\frac{1}{2} \sigma^{2}
$$

and our forecast of the inflation rate is

$$
E\left(i_{T-\Delta, T} \mid \mathcal{F}_{t}\right)=\exp (\pi)-1 .
$$

Proof. Because $\log I_{T}=\log I_{t}+\left(\pi-\frac{1}{2} \sigma^{2}\right)(T-t)+\sigma\left(W_{T}-W_{t}\right)$ and $\log I_{T-12 \Delta}=$ $\log I_{t}+\left(\pi-\frac{1}{2} \sigma^{2}\right)(T-12 \Delta-t)+\sigma\left(W_{T-12 \Delta}-W_{t}\right)$, our forecast of the log return $\pi_{T-12 \Delta, T}$ of the inflation index under the lognormal model is

$$
E\left(\pi_{T-\Delta, T} \mid \mathcal{F}_{t}\right)=\pi-\frac{1}{2} \sigma^{2}
$$

Our forecast of the inflation rate $i_{T-12 \Delta, T}$ under the lognormal model is

$$
\begin{aligned}
E\left(i_{T-\Delta, T} \mid \mathcal{F}_{t}\right) & =E\left(\exp \left\{\frac{1}{12 \Delta}\left(\pi-\frac{1}{2} \sigma^{2}\right) 12 \Delta+\frac{1}{12 \Delta} \frac{1}{2} \sigma^{2}\left(W_{T}-W_{T-12 \Delta}\right)\right\}-1 \mid \mathcal{F}_{t}\right) \\
& =\exp (\pi)-1
\end{aligned}
$$




\subsection{Lognormal Model with Local Drift Rate}

Lemma 4 Our forecast of the log return of the inflation index under the lognormal model with local drift rate is

$$
E\left(\pi_{T-12 \Delta, T} \mid \mathcal{F}_{t}\right)=\pi_{t}-\frac{1}{2} \sigma^{2}
$$

and our forecast of the inflation rate is

$$
E\left(i_{T-12 \Delta, T} \mid \mathcal{F}_{t}\right)=\exp \left\{\pi_{t}\right\}-1 \text {. }
$$

Proof. Because $\log I_{T}=\log I_{t}+\int_{t}^{T}\left(\pi_{s}-\frac{1}{2} \sigma^{2}\right) d s+\sigma\left(W_{T}-W_{t}\right)$, our forecast of the log return of the inflation index under the lognormal model with local drift rate is

$$
\begin{aligned}
E\left(\pi_{T-12 \Delta, T} \mid \mathcal{F}_{t}\right) & =\frac{1}{12 \Delta} E\left(\int_{T-12 \Delta}^{T}\left(\pi_{t}-\frac{1}{2} \sigma^{2}\right) d s+\sigma\left(W_{T}-W_{T-12 \Delta}\right) \mid \mathcal{F}_{t}\right) \\
& =\pi_{t}-\frac{1}{2} \sigma^{2}
\end{aligned}
$$

where we have assumed that the drift rate at time $t$ remains unchanged there-

after, based on information available at time $t$. Our forecast of the inflation rate is

$$
\begin{aligned}
E\left(i_{T-12 \Delta, T} \mid \mathcal{F}_{t}\right) & =E\left(\left(I_{T} / I_{T-12 \Delta}\right)^{1 /(12 \Delta)} \mathcal{F}_{t}\right)-1 \\
& =E\left(\exp \left\{\pi_{t}-\frac{1}{2} \sigma^{2}+\sigma\left(W_{T}-W_{T-12 \Delta}\right)\right\}\right)-1 \\
& =\exp \left\{\pi_{t}\right\}-1 .
\end{aligned}
$$

\subsection{Square Root Model}

Theorem 5 Our forecast of the log return of the inflation index under the square root model is

$$
E\left(\pi_{T-12 \Delta, T} \mid \mathcal{F}_{t}\right)=\frac{1}{12 \Delta}\left\{E\left[\log I_{T} \mid \mathcal{F}_{t}\right]-E\left[\log I_{T-12 \Delta} \mid \mathcal{F}_{t}\right]\right\}
$$

where the expected logarithms are computed using the approximation

$$
E\left[\log I_{T} \mid \mathcal{F}_{t}\right] \approx \log I_{t}-\log \lambda_{t, T}-\log \frac{4+\lambda_{t, T}}{8+4 \lambda_{t, T}}+\psi\left(\frac{\left(4+\lambda_{t, T}\right)^{2}}{8+4 \lambda_{t, T}}\right)
$$

where $\lambda_{t, T}=I_{t} /\left(\varphi_{T}-\varphi_{t}\right)$ and where $\phi(\alpha)=\phi^{\prime}(\alpha) / \phi(\alpha)$ is the digamma function.

Our forecast of the inflation rate is

$$
E\left[i_{T-12 \Delta, T} \mid \mathcal{F}_{t}\right)=4 \frac{\varphi_{T}-\varphi_{T-12 \Delta}}{I_{t}}\left(1-\exp \left(-\lambda_{t, T-12 \Delta} / 2\right)\right) .
$$


Proof. Under the square root model, the conditional random variable $I_{T} /\left(\varphi_{T}-\right.$ $\left.\varphi_{t}\right)$, based on information as at time $t$, has a non-central chi-squared distribution with four degrees of freedom and non-centrality parameter $\lambda_{t, T}=I_{t} /\left(\varphi_{T}-\varphi_{t}\right)$, where $\varphi_{t}$ is given in (6). We know that for a gamma-distributed random variable $Y \sim \operatorname{Gamma}(\alpha, \gamma)$, the mean is $\alpha / \gamma$, the variance is $\alpha / \gamma^{2}$ and its expected logarithm is given by

$E[\log Y]=\frac{\lambda^{\alpha}}{\Gamma(\alpha)} \int_{0}^{\infty} \log (y) y^{\alpha-1} \exp (-\gamma y) d y=-\log \gamma+\frac{\Gamma^{\prime}(\alpha)}{\Gamma(\alpha)} \equiv-\log \gamma+\psi(\alpha)$,

where $\psi$ is the digamma function. Approximating the non-central chi-squared random variable, which has mean $4+\lambda$ and variance $8+4 \lambda$, by a gammadistributed random variable having the same mean and variance, we arrive at the approximation

$$
E\left[\log \left\{I_{T} /\left(\varphi_{T}-\varphi_{t}\right)\right\} \mid \mathcal{F}_{t}\right] \approx-\log \frac{4+\lambda_{t, T}}{8+4 \lambda_{t, T}}+\psi\left(\frac{\left(4+\lambda_{t, T}\right)^{2}}{8+4 \lambda_{t, T}}\right) .
$$

Therefore,

$$
\begin{aligned}
E\left[\log I_{T} \mid \mathcal{F}_{t}\right] & =\log \left(\varphi_{T}-\varphi_{t}\right)+E\left[\log I_{T} /\left(\varphi_{T}-\varphi_{t}\right) \mid \mathcal{F}_{t}\right] \\
& \approx \log I_{t}-\log \lambda_{t, T}-\log \frac{4+\lambda_{t, T}}{8+4 \lambda_{t, T}}+\psi\left(\frac{\left(4+\lambda_{t, T}\right)^{2}}{8+4 \lambda_{t, T}}\right)
\end{aligned}
$$

and our forecast of the log return of the inflation index under the square root model is

$$
E\left(\pi_{T-12 \Delta, T} \mid \mathcal{F}_{t}\right)=\frac{1}{12 \Delta}\left\{E\left[\log I_{T} \mid \mathcal{F}_{t}\right]-E\left[\log I_{T-12 \Delta} \mid \mathcal{F}_{t}\right]\right\}
$$

where the expected logarithms are computed using (49).

Our forecast of the inflation rate is given by

$$
\begin{aligned}
& E\left[\left(I_{T} / I_{T-12 \Delta}\right)^{1 /(12 \Delta)}-1 \mid \mathcal{F}_{t}\right] \\
& =E\left[E\left[\left(I_{T} / I_{T-12 \Delta}\right)^{1 /(12 \Delta)} \mid \mathcal{F}_{T-12 \Delta}\right] \mid \mathcal{F}_{t}\right]-1 \\
& =E\left[E\left[I_{T} \mid \mathcal{F}_{T-12 \Delta}\right] / / I_{T-12 \Delta} \mid \mathcal{F}_{t}\right]-1 \\
& =E\left[\left(\varphi_{T}-\varphi_{T-12 \Delta}\right)\left(4+\lambda_{T-12 \Delta, T}\right) / I_{T-12 \Delta} \mid \mathcal{F}_{t}\right]-1 \\
& =4\left(\varphi_{T}-\varphi_{T-12 \Delta}\right) E\left[1 / I_{T-12 \Delta} \mid \mathcal{F}_{t}\right] \\
& =4 \frac{\varphi_{T}-\varphi_{T-12 \Delta}}{\varphi_{T-12 \Delta}-\varphi_{t}} E\left[\left(\varphi_{T-12 \Delta}-\varphi_{t}\right) / I_{T-12 \Delta} \mid \mathcal{F}_{t}\right] .
\end{aligned}
$$

Because $\left(\varphi_{T-12 \Delta}-\varphi_{t}\right) / I_{T-12 \Delta}$ is the reciprocal of a non-central chi-squared random variable with four degrees of freedom and non-centrality parameter $\lambda_{t, T-12 \Delta}=I_{t} /\left(\varphi_{T-12 \Delta}-\varphi_{t}\right)$, we have

$$
E\left[i_{T-12 \Delta, T} \mid \mathcal{F}_{t}\right)=4 \frac{\phi_{T}-\phi_{T-12 \Delta}}{\phi_{T-12 \Delta}-\phi_{t}}\left(1-\exp \left(-\lambda_{t, T-12 \Delta} / 2\right)\right) / \lambda_{t, T-12 \Delta},
$$

which is our result. 


\section{$5.43 / 2$ Model}

Theorem 6 Our forecast of the log return of the inflation index under the $3 / 2$ model is

$$
E\left(\pi_{T-12 \Delta, T} \mid \mathcal{F}_{t}\right)=\frac{1}{12 \Delta}\left\{E\left[\log I_{T} \mid \mathcal{F}_{t}\right]-E\left[\log I_{T-12 \Delta} \mid \mathcal{F}_{t}\right]\right\},
$$

where the expected logarithms are computed using the approximation

$$
E\left[\log I_{T} \mid \mathcal{F}_{t}\right] \approx \log I_{t}+\log \lambda_{t, T}+\log \frac{4+\lambda_{t, T}}{8+4 \lambda_{t, T}}-\psi\left(\frac{\left(4+\lambda_{t, T}\right)^{2}}{8+4 \lambda_{t, T}}\right)
$$

and where $\lambda_{t, T}=\left(I_{t}\right)^{-1} /\left(\xi_{T}-\xi_{t}\right)$.

Our forecast of the inflation rate is

$$
E\left[i_{T-12 \Delta, T} \mid \mathcal{F}_{t}\right)=-\left(\frac{\xi_{T}-\xi_{T-12 \Delta}}{\xi_{T}-\xi_{t}}\right)^{2} \exp \left\{-1 /\left(2 I_{t}\left(\xi_{T}-\xi_{t}\right)\right\} .\right.
$$

Proof. For the log return forecast, the proof is similar to that given in Theorem 5. For the inflation rate forecast,

$$
\begin{aligned}
& E\left[i_{T-12 \Delta, T} \mid \mathcal{F}_{t}\right] \\
& =E\left[I_{T} / I_{T-12 \Delta} \mid \mathcal{F}_{t}\right]-1 \\
& =E\left[E\left[I_{T} / I_{T-12 \Delta} \mid \mathcal{F}_{T-12 \Delta}\right] \mid \mathcal{F}_{t}\right]-1 .
\end{aligned}
$$

Now

$$
\begin{aligned}
& E\left[I_{T} / I_{T-12 \Delta} \mid \mathcal{F}_{T-12 \Delta}\right] \\
& =\frac{1}{I_{T-12 \Delta}} E\left[I_{T} \mid \mathcal{F}_{T-12 \Delta}\right] \\
& =\frac{1}{I_{T-12 \Delta}}\left(\xi_{T}-\xi_{T-12 \Delta}\right)^{-1} E\left[\frac{\xi_{T}-\xi_{T-12 \Delta}}{J_{T}} \mid \mathcal{F}_{T-12 \Delta}\right] \\
& =\frac{1}{I_{T-12 \Delta}}\left(\xi_{T}-\xi_{T-12 \Delta}\right)^{-1}\left(1-\exp \left(-\frac{1}{2} \lambda_{T-12 \Delta, T}\right)\right) / \lambda_{T-12 \Delta, T} \\
& =1-\exp \left(-\frac{1}{2} \lambda_{T-12 \Delta, T}\right) .
\end{aligned}
$$

Thus,

$$
\begin{aligned}
& E\left[i_{T-12 \Delta, T} \mid \mathcal{F}_{t}\right] \\
& =E\left[1-\exp \left(-\frac{1}{2} \lambda_{T-12 \Delta, T}\right) \mid \mathcal{F}_{t}\right]-1 \\
& =-E\left[\exp \left(-\frac{1}{2} \lambda_{T-12 \Delta, T}\right) \mid \mathcal{F}_{t}\right] \\
& =-M G F_{\chi_{4, \lambda_{t, T-12 \Delta}}^{2}}\left(-\frac{1}{2} \frac{\xi_{T-12 \Delta}-\xi_{t}}{\xi_{T}-\xi_{T-12 \Delta}}\right) \\
& =-\left(\frac{\xi_{T}-\xi_{T-12 \Delta}}{\xi_{T}-\xi_{t}}\right)^{2} \exp \left\{-1 /\left(2 I_{t}\left(\xi_{T}-\xi_{t}\right)\right)\right\},
\end{aligned}
$$


where we have used the formula

$$
M G F_{\chi_{4, \lambda}^{2}}(t)=\frac{1}{(1-2 t)^{\nu / 2}} \exp \{\lambda t /(1-2 t)\}
$$

for the moment generating function of a non-central chi-squared random variable. For the case when $t=T-12 \Delta$,

$$
\begin{aligned}
& E\left[I_{T} / I_{T-12 \Delta}-1 \mid \mathcal{F}_{t}\right] \\
& =E\left[\frac{J_{t}}{J_{T}} \mid \mathcal{F}_{t}\right]-1 \\
& =1-\exp \left(-\frac{1}{2} \lambda_{t, T}\right)-1 \\
& =-\exp \left(-\frac{1}{2} \lambda_{t, T}\right) .
\end{aligned}
$$

\subsection{Vasicek Drift Rate}

Theorem 7 Our forecast of the log return of the inflation index under the Vasicek drift rate model is

$E\left(\pi_{T-12 \Delta, T} \mid \mathcal{F}_{t}\right)=\left(\pi_{t}-\bar{\pi}\right) \exp (-\kappa(T-12 \Delta-t)) \frac{1}{12 \kappa \Delta}\{1-\exp (-12 \kappa \Delta)\}+\bar{\pi}$

and our forecast of the inflation rate is

$$
E\left(i_{T-12 \Delta, T} \mid \mathcal{F}_{t}\right)=\exp \left\{E\left(\pi_{T-12 \Delta, T} \mid \mathcal{F}_{t}\right)+\frac{1}{2} V A R\left(\pi_{T-12 \Delta, T} \mid \mathcal{F}_{t}\right)\right\}-1 .
$$

Proof. Integrating the SDE in (13), we have

$$
\pi_{s}=\pi_{t} \exp (-\kappa(s-t))+\bar{\pi}(1-\exp (-\kappa(s-t)))+\omega \int_{t}^{s} \exp (-\kappa(s-u)) d Z_{u} .
$$

Because

$$
\log I_{T}=\log I_{t}+\int_{t}^{T} \pi_{s} d s
$$


we have

$$
\begin{aligned}
& \log \left(I_{T} / I_{T-12 \Delta}\right) \\
& =\int_{T-12 \Delta}^{T} \pi_{s} d s \\
& =\int_{T-12 \Delta}^{T}\left\{\pi_{t} \exp (-\kappa(s-t))+\bar{\pi}(1-\exp (-\kappa(s-t)))+\omega \int_{t}^{s} \exp (-\kappa(s-u)) d Z_{u}\right\} d s \\
& =\left(\pi_{t}-\bar{\pi}\right) \exp (-\kappa(T-12 \Delta-t)) \frac{1}{\kappa}(1-\exp (-12 \kappa \Delta))+12 \bar{\pi} \Delta \\
& +\omega \int_{t}^{T-12 \Delta}\left\{\int_{T-12 \Delta}^{T} \exp (-\kappa(s-u)) d s\right\} d Z_{u} \\
& +\omega \int_{T-12 \Delta}^{T}\left\{\int_{u}^{T} \exp (-\kappa(s-u)) d s\right\} d Z_{u} \\
& =\left(\pi_{t}-\bar{\pi}\right) \exp (-\kappa(T-12 \Delta-t)) \frac{1}{\kappa}(1-\exp (-12 \kappa \Delta))+12 \bar{\pi} \Delta \\
& +\omega \int_{t}^{T-12 \Delta} \frac{1}{\kappa}\{\exp (-\kappa(T-12 \Delta-u))-\exp (-\kappa(T-u))\} d Z_{u} \\
& +\omega \int_{T-12 \Delta}^{T} \frac{1}{\kappa}\{1-\exp (-\kappa(T-u))\} d Z_{u} .
\end{aligned}
$$

The variance of $\log \left(I_{T} / I_{T-12 \Delta}\right)$ is

$$
\begin{aligned}
& V A R\left[\log \left(I_{T} / I_{T-12 \Delta}\right)\right] \\
& =\omega^{2} \int_{t}^{T-12 \Delta} \frac{1}{\kappa^{2}}[\{\exp (-\kappa(T-12 \Delta-u))-\exp (-\kappa(T-u))\}]^{2} d u \\
& +\omega^{2} \int_{T-12 \Delta}^{T} \frac{1}{\kappa^{2}}[\{1-\exp (-\kappa(T-u))\}]^{2} d u,
\end{aligned}
$$

which simplifies as

$$
\begin{aligned}
& \frac{\omega^{2}}{\kappa^{2}} \int_{t}^{T-12 \Delta} \exp (-2 \kappa(T-12 \Delta-u))\{1-\exp (-12 \kappa \Delta)\}^{2} d u \\
& +\frac{\omega^{2}}{\kappa^{2}} \int_{T-12 \Delta}^{T}\{1-2 \exp (-\kappa(T-u))+\exp (-2 \kappa(T-u))\} d u \\
& =\frac{\omega^{2}\{1-\exp (-12 \kappa \Delta)\}^{2}}{2 \kappa^{3}}\{1-\exp (-2 \kappa(T-12 \Delta-t))\} \\
& +\frac{\omega^{2}}{\kappa^{2}}\{12 \Delta-2(1-\exp (-12 \kappa \Delta)) / \kappa+(1-\exp (-24 \kappa \Delta)) / \kappa\} \\
& =\frac{\omega^{2}\{1-\exp (-12 \kappa \Delta)\}^{2}}{2 \kappa^{3}}\{1-\exp (-2 \kappa(T-12 \Delta-t))\} \\
& +\frac{\omega^{2}}{\kappa^{2}}\left\{1-(1-\exp (-12 \kappa \Delta))^{2} / \kappa\right\} .
\end{aligned}
$$


Therefore, our forecast of the log return of the inflation index is

$$
\begin{aligned}
E\left(\pi_{T-12 \Delta, T} \mid \mathcal{F}_{t}\right) & =\frac{1}{12 \Delta} E\left[\log \left(I_{T} / I_{T-12 \Delta}\right) \mid \mathcal{F}_{t}\right] \\
& =\left(\pi_{t}-\bar{\pi}\right) \exp (-\kappa(T-12 \Delta-t)) \frac{1}{12 \kappa \Delta}\{1-\exp (-12 \kappa \Delta)\}+\bar{\pi}
\end{aligned}
$$

and our forecast of the inflation rate is

$$
E\left(i_{T-12 \Delta, T} \mid \mathcal{F}_{t}\right)=\exp \left\{E\left(\pi_{T-12 \Delta, T} \mid \mathcal{F}_{t}\right)+\frac{1}{2} V A R\left(\pi_{T-12 \Delta, T} \mid \mathcal{F}_{t}\right)\right\}-1 .
$$

\subsection{Lognormal Model with Vasicek Drift Rate}

Theorem 8 Our forecast of the log return of the inflation index under the lognormal model with Vasicek drift rate is

$E\left(\pi_{T-12 \Delta, T} \mid \mathcal{F}_{t}\right)=\left(\pi_{t}-\bar{\pi}\right) \exp (-\kappa(T-12 \Delta-t)) \frac{1}{12 \kappa \Delta}\{1-\exp (-12 \kappa \Delta)\}+\bar{\pi}-\frac{1}{2} \sigma^{2}$

and our forecast of the inflation rate is

$$
E\left(i_{T-12 \Delta, T} \mid \mathcal{F}_{t}\right)=\exp \left\{E\left(\pi_{T-12 \Delta, T} \mid \mathcal{F}_{t}\right)+\frac{1}{2} V A R\left(\pi_{T-12 \Delta, T} \mid \mathcal{F}_{t}\right)\right\}-1 .
$$

Proof. The proof of the forecast of the log return is analogous to that given in the proof of Theorem 7. Because

$$
\log I_{T}=\log I_{t}+\int_{t}^{T} \pi_{s}-\frac{1}{2} \sigma^{2} d s+\sigma \int_{t}^{T} d W_{s}
$$

we have

$$
\begin{aligned}
& \log \left(I_{T} / I_{T-12 \Delta}\right) \\
& =\left(\pi_{t}-\bar{\pi}\right) \exp (-\kappa(T-12 \Delta-t)) \frac{1}{\kappa}(1-\exp (-12 \kappa \Delta))+12 \bar{\pi} \Delta-\frac{1}{2} \sigma^{2} 12 \Delta \\
& +\omega \int_{t}^{T-12 \Delta} \frac{1}{\kappa}\{\exp (-\kappa(T-12 \Delta-u))-\exp (-\kappa(T-u))\} d Z_{u} \\
& +\omega \int_{T-12 \Delta}^{T} \frac{1}{\kappa}\{1-\exp (-\kappa(T-u))\} d Z_{u}+\sigma \int_{T-12 \Delta}^{T} d W_{u} .
\end{aligned}
$$

The variance of $\log \left(I_{T} / I_{T-12 \Delta}\right)$ is

$$
\begin{aligned}
& V A R\left[\log \left(I_{T} / I_{T-12 \Delta}\right)\right] \\
& =\frac{\omega^{2}\{1-\exp (-12 \kappa \Delta)\}^{2}}{2 \kappa^{3}}\{1-\exp (-2 \kappa(T-12 \Delta-t))\} \\
& +\frac{\omega^{2}}{\kappa^{2}}\left\{1-(1-\exp (-12 \kappa \Delta))^{2} / \kappa\right\}+\sigma^{2} 12 \Delta .
\end{aligned}
$$




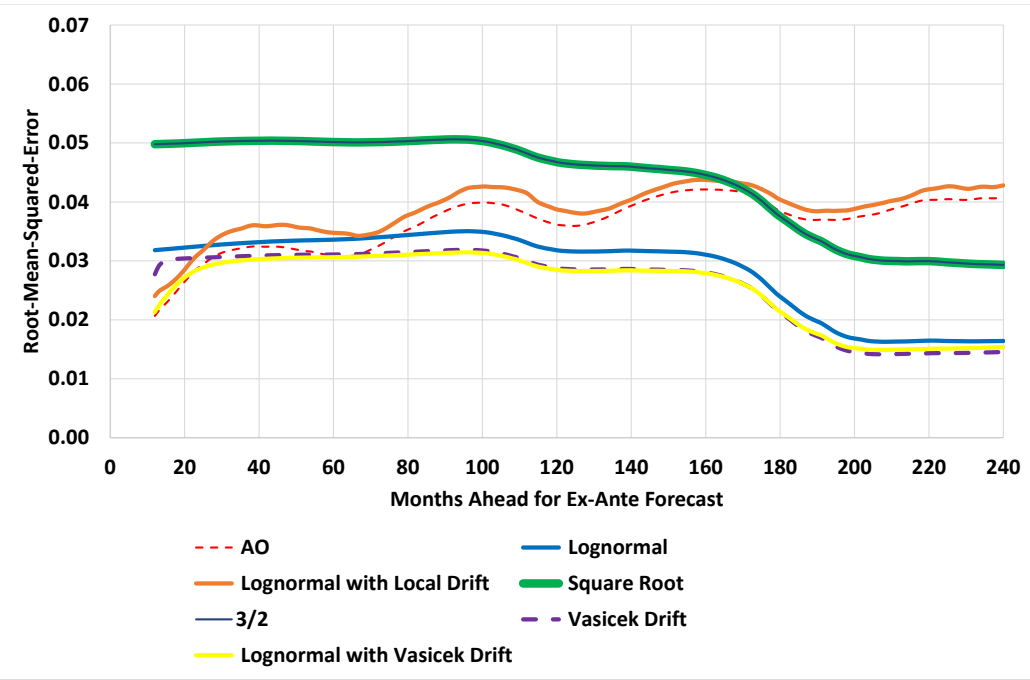

Figure 7: RMSEs of Forecasts.

Therefore, our forecast of the log return of the inflation index is

$$
\begin{aligned}
E\left(\pi_{T-12 \Delta, T} \mid \mathcal{F}_{t}\right) & =\frac{1}{12 \Delta} E\left[\log \left(I_{T} / I_{T-12 \Delta}\right) \mid \mathcal{F}_{t}\right] \\
& =\left(\pi_{t}-\bar{\pi}\right) \exp (-\kappa(T-12 \Delta-t)) \frac{1}{12 \kappa \Delta}\{1-\exp (-12 \kappa \Delta)\}+\bar{\pi}
\end{aligned}
$$

and our forecast of the inflation rate is

$$
E\left(i_{T-12 \Delta, T} \mid \mathcal{F}_{t}\right)=\exp \left\{E\left(\pi_{T-12 \Delta, T} \mid \mathcal{F}_{t}\right)+\frac{1}{2} V A R\left(\pi_{T-12 \Delta, T} \mid \mathcal{F}_{t}\right)\right\}-1 .
$$

\section{Conclusion}

We have investigated several continuous-time models of the inflation rate. It is evident that the lognormal model of the inflation index with Vasicek drift rate dynamics provides the best forecasts of annual inflation rates, when using the RMSE as the performance measure. In light of the improvement in the loglikelihood, AIC and BIC values shown in Table 1 for the $3 / 2$ model, as given in (8), over those for the lognormal and square root models, as given in (1) and (4), it would be worthwhile in future work investigating the forecasting performance of the $3 / 2$ model having Vasicek drift rate. 


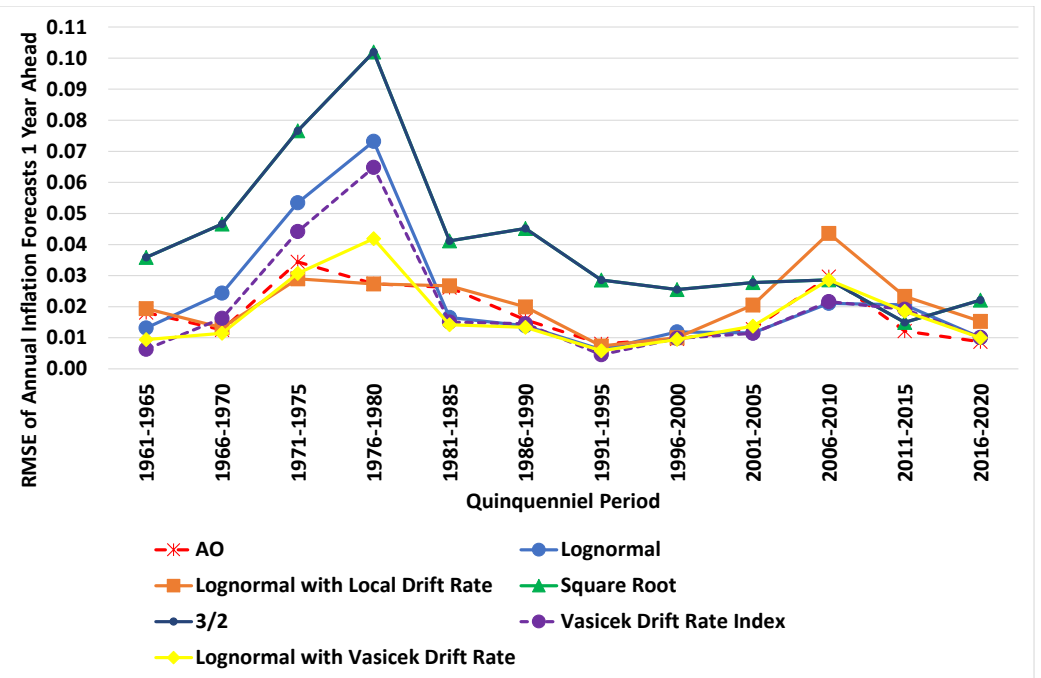

Figure 8: RMSEs of forecasts for each half decade over the past fifty years.

\section{References}

D.H. Ahn and B. Gao. A parametric nonlinear model of term structure dynamics. Review of Financial Studies, 12:721-762, 1999.

A. Atkeson and L. E. Ohanian. Are Phillips Curves useful for forecasting inflation? Federal Reserve Bank of Minneapolis Quarterly Review, 25(1):2-11, 2001.

A. R. Bergstrom. Continuous time stochastic models and issues of aggregation over time. In Z. Griliches and M. D. Intriligator, editors, Handbook of Econometrics, volume 2, pages 1145-1212. North Holland, 1984.

C. Chiarella, C. Hsiao, and W. Semmler. Intertemporal investment strategies under inflation risk. University of Technology, Sydney, Quantitative Finance Research Centre Research Paper 192, 2007.

J. C. Cox, J. E. Ingersoll, and S. A. Ross. A theory of the term structure of interest rates. Econometrica, 53(2):385-407, 1985a.

J. C. Cox, J. E. Ingersoll, and S. A. Ross. An intertemporal general equilibrium model of asset prices. Econometrica, 53(2):363-384, 1985b. 


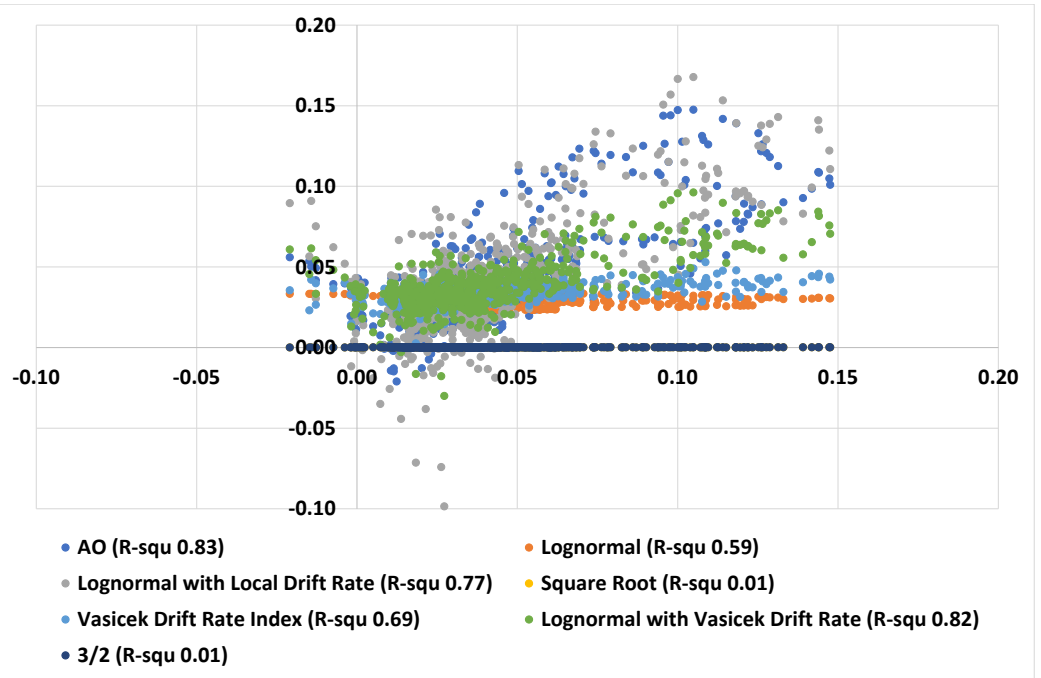

Figure 9: Scatter plot of model forecasts versus actual one-year inflation rate over the past fifty years. 
K. Fergusson. Explicit formulae for parameters of stochastic models of a discounted equity index using maximum likelihood estimation with applications. Annals of Financial Economics, 12(2):1-31, 2017. doi: 10.1142/S2010495217500105.

K. Fergusson and E. Platen. Application of maximum likelihood estimation to stochastic short rate models. Annals of Financial Economics, 10(2), 2015.

R. Jarrow and Y. Yildirim. Pricing treasury inflation protected securities and related derivatives using an HJM model. Journal of Finance and Quantitative Analysis, 38(2):337-358, 2003.

B. Meyer and M. Pasaogullari. Simple ways to forecast inflation: What works best? Economic Commentary, Federal Reserve Bank of Cleveland, Research Department, 2010.

E. Platen. A short-term interest rate model. Finance and Stochastics, 3:215-225, 1999.

E. Platen. A minimal financial market model. In Trends in Mathematics, pages 293-301. Birkhäuser, 2001.

J. H. Stock and M. W. Watson. Why has U.S. inflation become harder to forecast? Journal of Money, Credit and Banking, 39(1):3-34, 2007.

O. A. Vasicek. An equilibrium characterization of the term structure. Journal of Financial Economics, 5:177-188, 1977. 